\title{
STUDY OF FLUX EMITTED FROM THE OUTER SURFACE OF STAR SIRIUS OBSERVED FROM NATIONAL OBSERVATORY NAGARKOT, NEPAL
}

\author{
B.P. Pant*, P.R. Dhungel*, U. Khanal** and S.R. Shahi*** \\ *St.xavier's College Maitighar, Kathmandu, Nepal. \\ **Central Department of Physics, Tribhuvan university, Kirtipur, Kathmandu, Nepal. \\ ***B.P. Koiral Memorial Planetarium, Observataroy and Science Museum Development Board, Singha Dubar, \\ Kathmandu, Nepal.
}

\begin{abstract}
We present the 'Study of flux emitted from the outer surface of star Sirius'. The FITS images were collected by Meade 16 inch LX200GPS Schmidt-Cassegrain Telescope during the period from December 2011 to November 2012 from the National Observatory Nagarkot, Nepal. The images captured in FITS (Flexible Image Transport System) format using AUTOSTAR SUITE 3.08 software and was processed in the software ALADIN 2.5. The data so obtained were analyzed in ORIGIN 5.0. The observed two dimensional image of star was divided into 12 direction and variation of relative density along each direction was measured. It was found that there was consistent decrease in relative flux density from inner to outer region. The relative flux density per pixel was found in the range of 84.17 to 116.75 . In addition, we studied the outer contour of all regions (i.e., four quadrants). The value of relative flux density for first, second, third and fourth region were found to be $23.17,23.56,23.16$ and 23.10 respectively. This suggest that the structure of Sirius was symmetrical and no significant flatting of disk was observed from our analysis.
\end{abstract}

Keywords: Sirius ; Observation; Schmidt-Cassegrain Telescope; Flux density.

\section{INTRODUCTION}

Sirius (HD 48915, HIP 32349, G 66) is white mainsequence star of spectral type A1V, termed Sirius A, With a visual apparent magnitude of $-1.44,<\mathrm{U}-\mathrm{B}>$ color index -0.05 and $\langle\mathrm{B}-\mathrm{V}\rangle$ color index 0.01 , it is almost twice as bright as Canopus, the next brightest star and a faint white dwarf companion of spectral type DA2, called Sirius B.

Fig 1: FITS image of Sirius taken on November 09,2012
The star has the Bayer designation Alpha Cains Majoris $(\alpha \mathrm{CMa})$. Sirius appears bright because of both its intrinsic luminosity and its proximity to Earth. At a distance of 2.6 parsecs $(8.48 \mathrm{ly})$, as determined by the Hipparcos astrometry satellite ${ }^{[1]}$, the Sirius system is one of Earth's near neighbors; for Northern-hemisphere observers between 30 degrees and 73 degrees of latitude, it is the closest star (after the Sun) that can be seen with a naked eye. Sirius is gradually moving closer to the Solar System, so it will slightly increase in brightness over the next 60,000 years. After that time its distance will begin to recede, but it will continue to be the brightest star in the Earth's sky for the next 210,000 years [2] The system is between 200 and 300 million years old. It was originally composed of two bright bluish stars. The distance separating Sirius A from its companion varies between 8.1 and $31.5 \mathrm{AU}^{[3]}$. The more massive of these, Sirius B, consumed its resources and became a red giant before shedding its outer layers and collapsing into its current state as a white dwarf around 120 million years ago. The projected rotational velocity is a relatively low $16 \mathrm{~km} / \mathrm{s}^{[4]}$, which does not produce any significant flatting of its disk ${ }^{[5]}$. Thus, we took it as an object of interest and decided to study its symmetrical feature by processing FITS image obtained from the National Observatory through the data reduction software ALADIN2.5.

Author for Correspondence: B.P. Pant St.xavier's College Maitighar, Kathmandu, Nepal. E-mail: ptbiren@gmail.com. 


\section{OBSERVATION}

Sirius was observed through Meade 16 inch LX200GPS telescope installed at the National Observatory, Nagarkot during the period from December 2011 to November 2012 .

\section{OBSERVATION TECHNIQUES}

A Google Sky Map and star chart is used to identify the heavenly bodies and there actual position in the sky with naked eyes. Before observing a particular heavenly body (one of the brightest star, Sirius), we identified Deneb of Cygnus constellation and it is defined as the reference frame with the telescope at its center while the Altair is taken as another reference frame. We manually set the telescope so that the Altair was at the center of the field of view. After this star was set in the memory of the telescope, we set it again to focus on the Deneb. When this star was set in the memory, we got the message "Alignment Successful". This message ensured that the reference frame was set and we were ready to use the telescope to view any possible object in the sky. Once a reference frame is set, the telescope works automatically as per the commands given to it through the AUTOSTAR II hand box. When we give the command to go to the Sirius star, the telescope would rotate itself to focus on it. The star would appear in or near the field of view, depending on the accuracy with which we fix a reference frame. Once the telescope aligns itself to focus on the desired object, the only effort we have to make is to bring it in the center of the field of view. When the brightest star Sirius is in the field of view of eyepiece we removed the eyepiece and used cable which allows to show image on screen, from where we are able to capture FITS and JPEJ image of Sirius star.

\section{Graphical Analysis and Data Reduction}

Sirius's photographic images obtained by telescope is a two dimensional projection of the distant three dimensional structure. FITS image is proceed with the data reduction software ALADIN 2.5 to generate information about the value of relative flux density (flux) at each pixel. We decided to study the variation of relative flux density along different directions and in different regions. We drew 12 lines that meet at the center such that angle between any two adjacent lines is almost $30^{\circ}$. We clicked on each pixel from the inner to the outer region that the lines pass through. We recorded the position and relative flux density associated with each of the clicked pixel. The further processing was done in ORIGIN 5.0. ORIGIN is an easy-to-use software application developed by Microcal Software, Inc. It provides powerful data analysis, curvefitting capabilities, and publication-quality graphing capabilities. Using this software, we plotted graphs for relative flux density against number of pixel and obtained their polynomial fits.

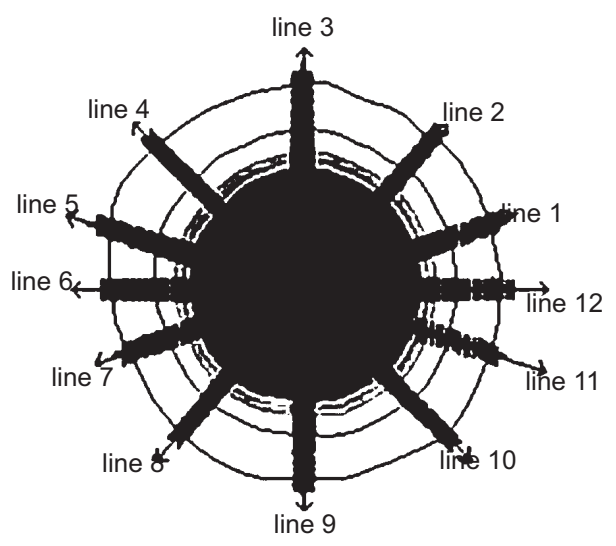

Fig 2: image of the Sirius showing contours, axes and clicks, i.e., clicks along twelve lines in the software ALADIN 2.5

\section{RESULT AND DISCUSSION}

\section{Relative Flux Density}

We give the results concerning the variation of relative flux density in and around the star Sirius. For this we used the image captured by us using Meade 16-inch LX200GPS Schmidt-Cassegrain telescope at Nagarkot Observatory during November, 2012. Our prime interest is to study the variation of relative flux density around the star Sirius.

\section{Relative Flux Density Variation from the Inner to the Outer Region}

In this section we are going to discuss the variation of relative flux density along the 12 lines from the inner to the outer region. The values of the relative flux density for these lines have been obtained from ALADIN2.5 while the associated graphs have been derived from ORIGIN5.0 software.

\section{Variation of Relative Flux density along line One}

Now we are going to discuss the values of relative flux density for line 1 which lies towards east in the $1^{\text {st }}$ quadrant (i.e., at an angle of $30^{\circ}$ with the horizontal axis). The values are listed on the table 1 .

The first column gives the serial number for the clicks (i.e., pixels) showing that the clicks are from inner to outer region, the second and third columns give the pixel coordinates, the fourth column gives the value of relative flux density for each pixel, and the last column gives standard error in flux density i.e., $\mathrm{F}_{\mathrm{D}(\mathrm{Er} \pm) \text {. }}$ 
Table 1: The values of relative flux density along line one

\begin{tabular}{|c|c|c|c|c|}
\hline$P_{N}$ & $\mathrm{x}$ & Y & $\mathrm{F}_{\mathrm{D}}$ & $\mathrm{F}_{\mathrm{D}(\mathrm{Er} \pm)}$ \\
\hline 1 & 205.59 & 160.06 & 255.04 & 12.75 \\
\hline 2 & 205.93 & 159.93 & 254.62 & 12.73 \\
\hline 3 & 206.21 & 159.87 & 250.62 & 12.53 \\
\hline 4 & 206.59 & 159.75 & 250.62 & 12.53 \\
\hline 5 & 206.92 & 159.62 & 236.75 & 11.83 \\
\hline 6 & 207.18 & 159.53 & 236.75 & 11.83 \\
\hline 7 & 208.21 & 159.12 & 201.62 & 10.08 \\
\hline 8 & 208.53 & 159.03 & 201.62 & 10.08 \\
\hline 9 & 208.81 & 158.87 & 184.37 & 9.21 \\
\hline 10 & 209.21 & 158.71 & 150.03 & 7.50 \\
\hline 11 & 210.09 & 158.37 & 121.75 & 6.08 \\
\hline 12 & 211.06 & 157.96 & 95.25 & 4.76 \\
\hline 13 & 211.96 & 157.68 & 80.02 & 4.00 \\
\hline 14 & 212.96 & 157.28 & 66.37 & 3.31 \\
\hline 15 & 213.91 & 156.93 & 60.87 & 3.04 \\
\hline 16 & 214.15 & 156.84 & 51.62 & 2.85 \\
\hline 17 & 214.43 & 156.75 & 51.62 & 2.85 \\
\hline 18 & 215.68 & 156.21 & 45.37 & 2.26 \\
\hline 19 & 215.87 & 156.15 & 45.37 & 2.26 \\
\hline 20 & 216.06 & 156.12 & 40.02 & 2.00 \\
\hline 21 & 216.31 & 155.93 & 38.52 & 1.92 \\
\hline 22 & 216.62 & 155.87 & 38.51 & 1.92 \\
\hline 23 & 217.34 & 155.62 & 34.37 & 1.71 \\
\hline 24 & 218.04 & 155.34 & 30.12 & 1.50 \\
\hline 25 & 219.03 & 154.96 & 24.37 & 1.21 \\
\hline 26 & 219.43 & 154.81 & 24.37 & 1.21 \\
\hline 27 & 219.78 & 154.65 & 24.37 & 1.21 \\
\hline 28 & 220.06 & 154.56 & 21.87 & 1.09 \\
\hline 29 & 220.41 & 154.43 & 21.87 & 1.09 \\
\hline 30 & 221.04 & 154.21 & 19.25 & 0.96 \\
\hline 31 & 221.25 & 154.09 & 19.25 & 0.96 \\
\hline 32 & 221.53 & 153.96 & 19.02 & 0.95 \\
\hline 33 & 221.84 & 153.91 & 19.01 & 0.95 \\
\hline 34 & 222.06 & 153.81 & 17.12 & 0.85 \\
\hline 35 & 222.28 & 153.71 & 17.12 & 0.85 \\
\hline 36 & 222.53 & 153.59 & 17.12 & 0.85 \\
\hline 37 & 222.78 & 153.54 & 17.12 & 0.85 \\
\hline
\end{tabular}

The variation of relative flux density along the line one is shown in figure 3. The graphs are plotted pixel number versus relative flux density. The graphs show that relative flux density continuously decreases from inner to outer regions.

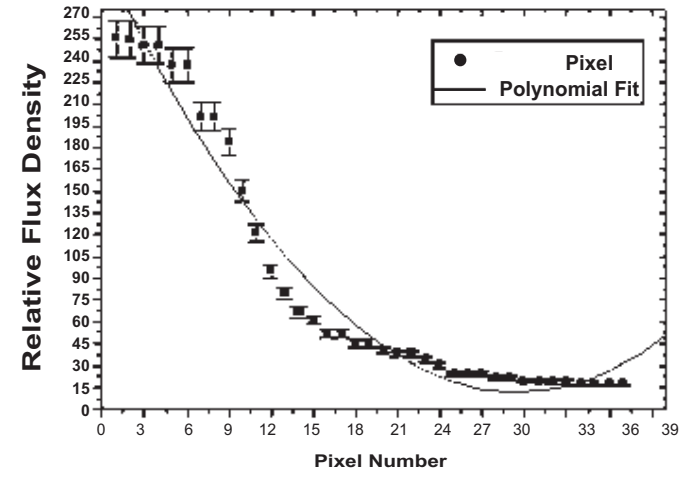

Fig 3: Variation of relative flux density along line one.

We took the first column of Table 1 to ORIGIN 5.0 and labeled with pixel number i.e. ' $r$ '. We took the fourth column to ORIGIN 5.0 and labeled it with relative flux density $\left(\mathrm{F}_{\mathrm{D}}\right)$. We calculated the standard error for the column of relative flux density $\left(\mathrm{F}_{\mathrm{D}}\right)$ ( i.e., for column four) and plotted the graph of relative flux density against $r$. As the value of $r$ increases, the value of relative flux density goes on decreasing so that the slope goes on changing. In each of the graphs, square represents values of relative flux densities corresponding to values of r. Each of the squares is attached to two bars, one on its upper side and the other on its lower side. These bars are called error bars. The error is actually standard error for the values of relative flux density as obtained through standard analysis. The nature of relationship between relative flux density and pixel number is determined by using parabolic fit model $y=a_{1} x^{2}+b_{1} x$ $+c_{1}$. The curve fitting of relative flux density against pixel number for line one is shown in Fig.3, where a solid curve represents second order polynomial fit. For the first line, the second order polynomial is found to be $y=0.34 x^{2}-20.24 x+310.36$ with error in the coefficients $0.02,1.07,8.87$ respectively. The standard deviation $(\sigma)$ for the second order polynomial model is 17.03. While comparing standard error with standard deviation obtained from second order polynomial fit, standard error is less than standard deviation which shows that the relative flux density distribution has no significant deviation from the polynomial fit.

\section{Flux Variation on all twelve lines}

Table 2: Summary of the relative flux density along all 12 lines: The first two columns list the lines and the total number of clicks (i.e., pixels) along each line. The third column gives the total relative flux density. The last column represents the value of relative flux density per pixel. We can see that the value of relative flux density per pixel is maximum for line three and minimum for line six. 
Table 2 : Summary of the relative flux density along all 12 lines

\begin{tabular}{llll}
\hline Lines & $\mathrm{N}$ & $\sum \mathrm{F}_{\mathrm{D}}$ & $\mathrm{F}_{\mathrm{DP}}$ \\
1 & 37 & 3283.68 & 88.74 \\
2 & 38 & 4358.09 & 114.68 \\
3 & 55 & 6420.45 & 116.73 \\
4 & 37 & 3401.14 & 91.92 \\
5 & 35 & 3592.31 & 102.63 \\
6 & 31 & 2629.71 & 84.82 \\
7 & 37 & 3912.64 & 105.74 \\
8 & 39 & 3859.88 & 98.97 \\
9 & 45 & 4436.00 & 98.57 \\
10 & 38 & 3745.48 & 98.56 \\
11 & 37 & 3944.42 & 106.60 \\
12 & 37 & 4211.43 & 113.82 \\
\hline
\end{tabular}

\section{DISCUSSION}

We have already discussed the nature of the graph for line 1 . With regards to this graph and the information provided by the preceding tables (table 2), we can make a comparative analysis of the graphs for all other 11 lines.

Variation of relative flux density along line 2:

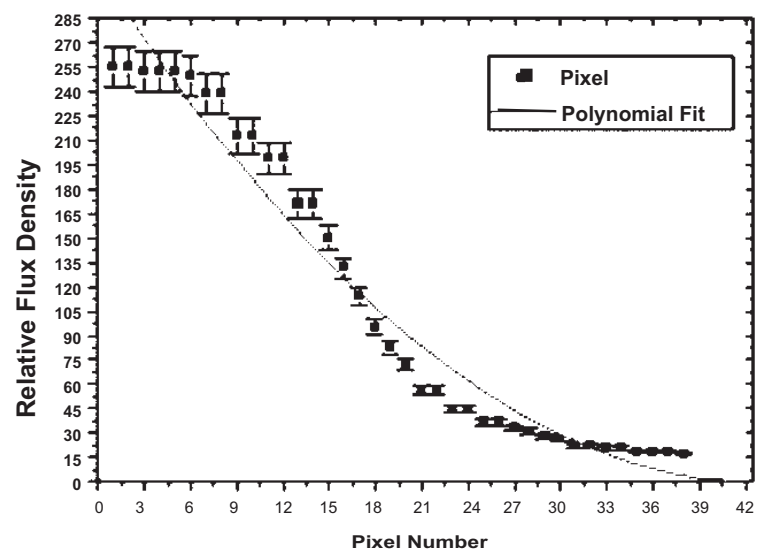

Fig 4: variation of relative flux density along line two

The variation of relative flux density along the line two (which lies an angle of $60^{\circ}$ from the horizontal axis) are shown in fig 4. The graphs are plotted pixel number i.e. $r$, versus relative flux density. The graphs show that relative flux density continually decreases from inner to outer regions. The nature of relationship between relative flux density and pixel number is determined by using parabolic fit model $y=a_{1} x^{2}+b_{1} x+c_{1}$. The curve fitting of relative flux density against pixel number for line two is shown in Fig. 4 where a solid curve represents second order polynomial fit. For the second line, the second order polynomial is found to be $\mathrm{y}=0.16 \mathrm{x}^{2}-14.60 \mathrm{x}+$ 314.79 with error in the coefficient $0.02,1.17,9.91$ respectively. The standard deviation $(\sigma)$ for the second order polynomial model is 19.30 .

Variation of relative flux density along line 3:

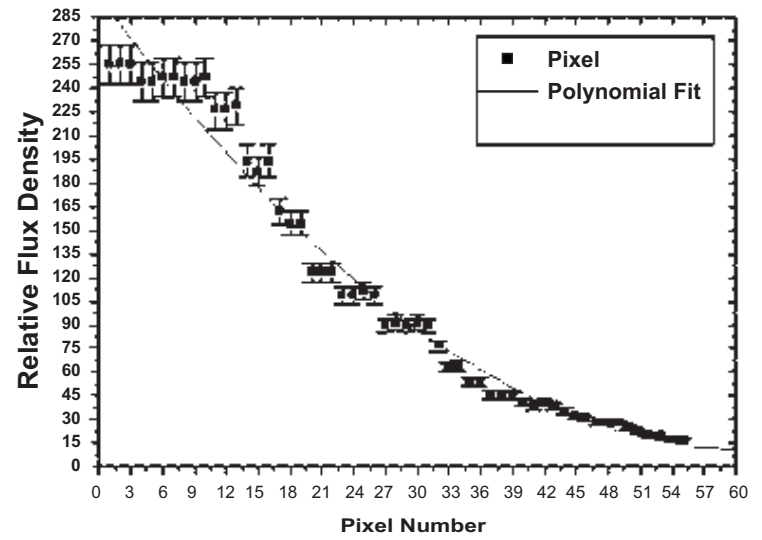

Fig 5: variation of relative flux density along line three

The variation of relative flux density along line three are shown in fig 5. This graph differs slightly from graph of line one and line two. The graph shows slightly variation of flux density in the region around pixel $r=9$. The nature of relationship between relative flux density and pixel number is determined by using parabolic fit model $y=a_{1} x^{2}+b_{1} x+c_{1}$. The curve fitting of relative flux density against pixel number for line three is shown in Fig. 5 where a solid curve represents second order polynomial fit. For the second line, the second order polynomial is found to be $y=0.07 x^{2}-9.40 x+299.89$ with error in the coefficient $0.007,0.43,5.52$ respectively. The standard deviation $(\sigma)$ for the second order polynomial model is 13.55 .

Variation of relative flux density along line 4:

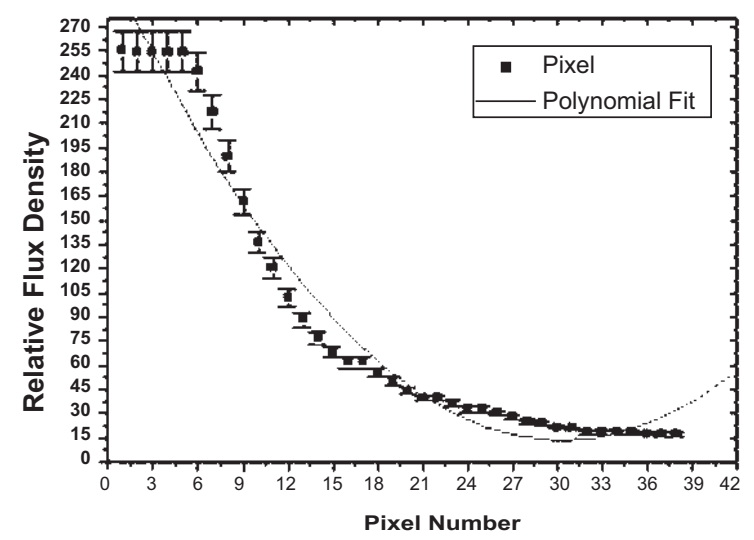

Fig 6: variation of relative flux density along line four

The variation of relative flux density along the line four (which lies in second quadrants) are shown in fig 6 (Symbols as in Fig 5.). The graphs show that relative flux density continually decreases from inner to outer regions. For the fourth line, the second order polynomial is found to be $\mathrm{y}=0.32 \mathrm{x}^{2}-19.80 \mathrm{x}+310.52$ with error in the coefficient $0.024,0.99,8.37$ respectively. The standard deviation $(\sigma)$ for the second order polynomial model is 16.30 . 
Variation of relative flux density along line 5:

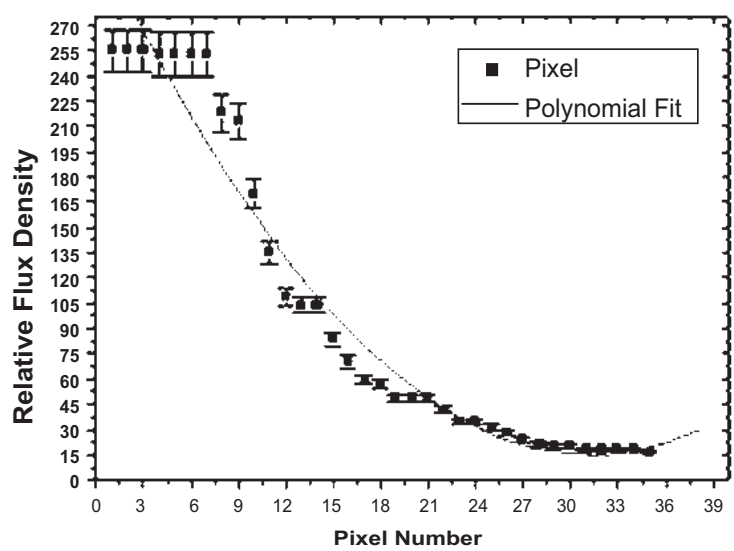

Fig 7: variation of relative flux density along line five

The variation of relative flux density along the line five is shown in figure 7 (Symbols as in Fig. 6). The graph shows that relative flux density continuously decreases from inner to outer regions. For the fifth line, the second order polynomial is found to be $\mathrm{y}=0.31 \mathrm{x}^{2}-19.68 \mathrm{x}+$ 322.84 with error in the coefficient $0.034,1.39,10.92$ respectively. The standard deviation $(\sigma)$ for the second order polynomial model is 20.32 .

Variation of relative flux density along line 6 :

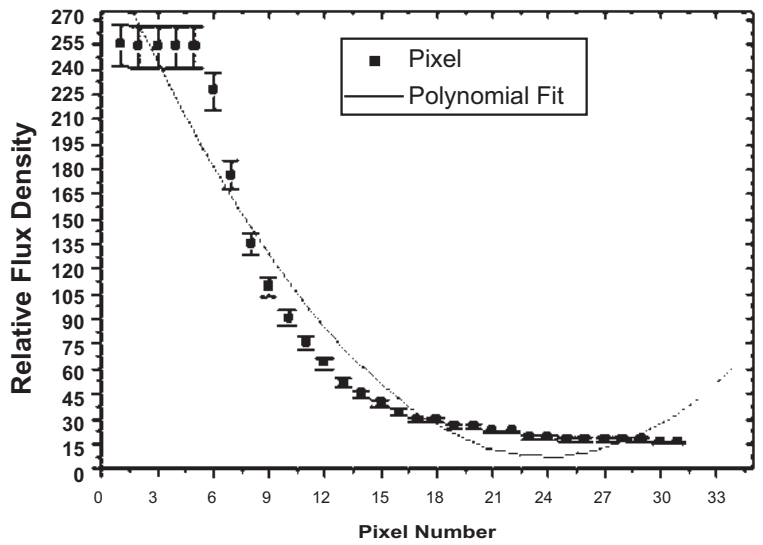

Fig 8: variation of relative flux density along line six

the variation of relative flux density along the line six is shown in figure 8 (Symbols as in Fig: 7). The graph shows similar nature as graph of line five. The graph shows that relative flux density continuously decreases from inner to outer regions. For the sixth line, the second order polynomial is found to be $\mathrm{y}=0.54 \mathrm{x}^{2}-26.10 \mathrm{x}+$ 319.25 with error in the coefficient $0.051,1.69,11.74$ respectively. The standard deviation $(\sigma)$ for the second order polynomial model is 20.41 .
Variation of relative flux density along line 7:

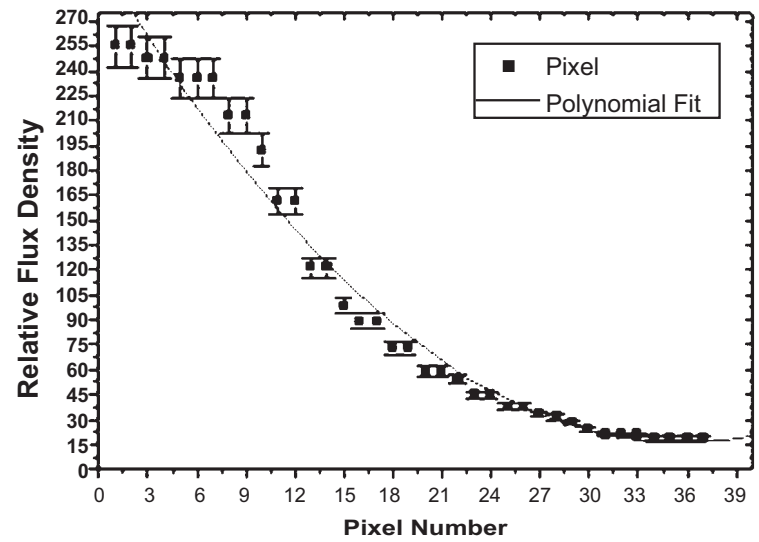

Fig 9: variation of relative flux density along line seven

The variation of relative flux density along the line seven is shown in figure 9 (Symbols as in Fig. 8). The graph shows similar nature as graph of line six. The graph shows that relative flux density continuously decreases from inner to outer regions. The second order polynomial is found to be $\mathrm{y}=0.22 \mathrm{x}^{2}-16.37 \mathrm{x}+$ 308.99 with error in the coefficient $0.023,0.91,7.56$ respectively. The standard deviation $(\sigma)$ for the second order polynomial model is 14.50 .

Variation of relative flux density along line 8:

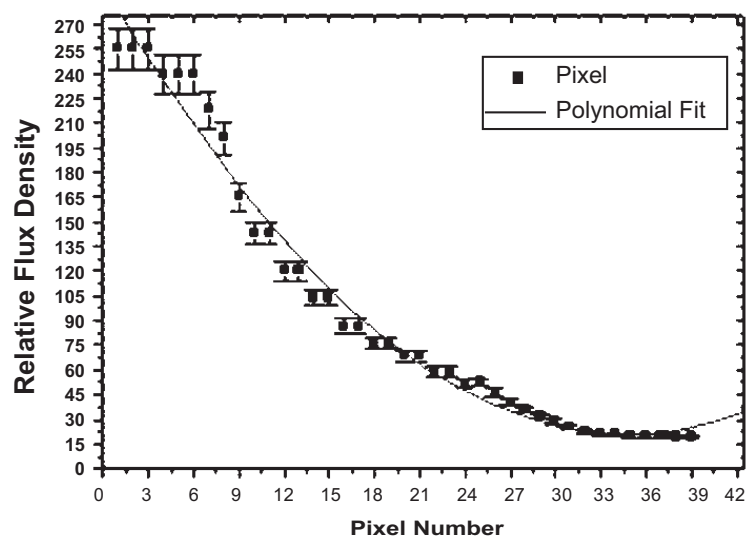

Fig 10: variation of relative flux density along line eight

The variation of relative flux density along the line eight (which lies in third quadrant) is shown in figure 10 (Symbols as in Fig. 9). The graph shows similar nature as graph of line seven. The graph shows that relative flux density continuously decreases from inner to outer regions. The second order polynomial is found to be $y$ $=0.22 \mathrm{x}^{2}-15.72 \mathrm{x}+294.53$ with error in the coefficient $0.015,0.65,5.66$ respectively. The standard deviation $(\sigma)$ for the second order polynomial model is 11.21. 
Variation of relative flux density along line 9:

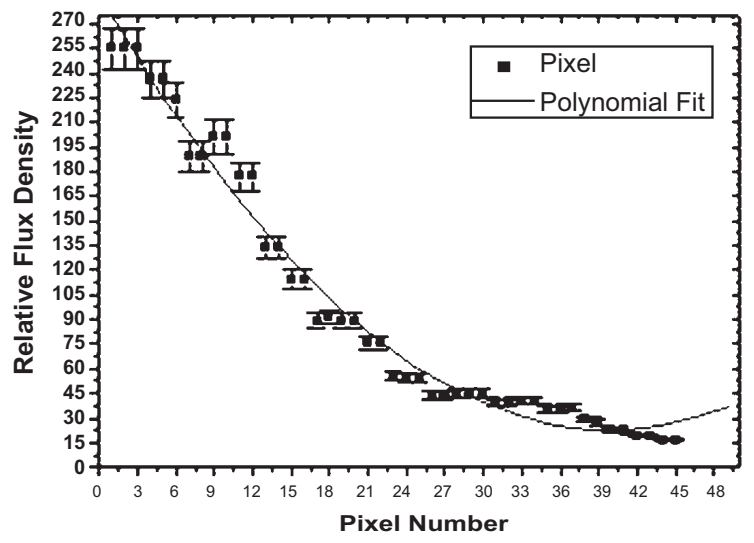

Fig 11: variation of relative flux density along line nine

The variation of relative flux density along the line Nine (which lies in third quadrant) is shown in figure 11 (Symbols as in Fig. 10). This graph is slightly differs from the other graphs it shows a slight variation of flux density in the region around pixel $=7$ and 29. Some in homogeneity is found in these region, The graph shows that relative flux density continuously decreases from inner to outer regions. The nature of relationship between relative flux density and pixel number is determined by using parabolic fit model $\mathrm{y}=\mathrm{a}_{1} \mathrm{x}^{2}+\mathrm{b}_{1} \mathrm{x}+\mathrm{c}_{1}$. The second order polynomial is found to be $\mathrm{y}=0.16 \mathrm{x}^{2}-13.27 \mathrm{x}+$ 288.02 with error in the coefficient $0.01,0.52,5.52$ respectively. The standard deviation $(\sigma)$ for the second order polynomial model is 11.75 .

Variation of relative flux density along line 10:

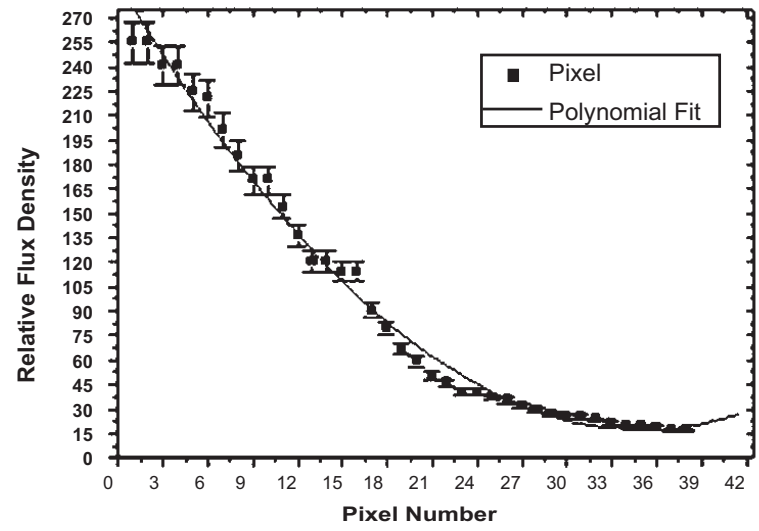

Fig 8: variation of relative flux density along line six

The variation of relative flux density along the line Ten (which lies in fourth quadrant) is shown in figure 12 (Symbols as in Fig. 11) The graph shows that relative flux density continuously decreases from inner to outer regions. The nature of relationship between relative flux density and pixel number is determined by using parabolic fit model $\mathrm{y}=\mathrm{a}_{1} \mathrm{x}^{2}+\mathrm{b}_{1} \mathrm{x}+\mathrm{c}_{1}$. The second order polynomial is found to be $\mathrm{y}=0.22 \mathrm{x}^{2}-15.61 \mathrm{x}+292.14$ with error in the coefficient $0.01,0.46,3.91$ respectively. The standard deviation $(\sigma)$ for the second order polynomial model is 7.62 .
Variation of relative flux density along line 11:

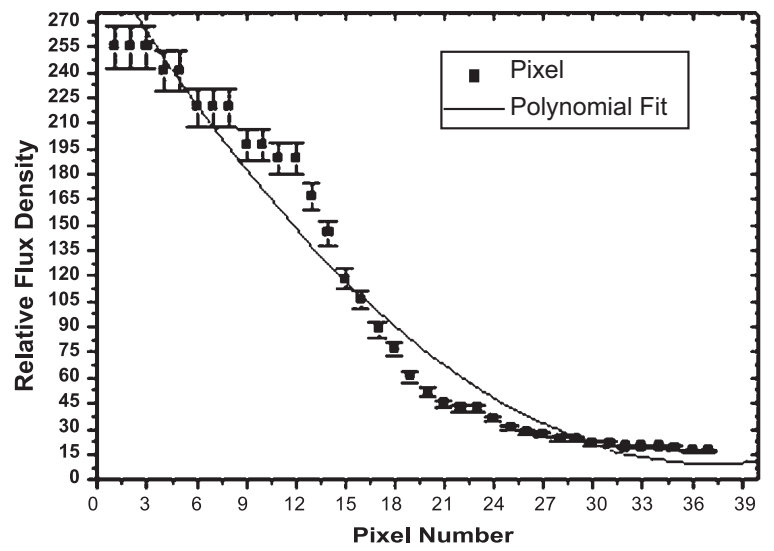

Fig 13: variation of relative flux density along line eleven

The variation of relative flux density along the line eleven (which lies in fourth quadrant) is shown in figure 13 (Symbols as in Fig. 12). The graph shows that relative flux density continuously decreases from inner to outer regions. The nature of relationship between relative flux density and pixel number is determined by using parabolic fit model $\mathrm{y}=\mathrm{a}_{1} \mathrm{x}^{2}+\mathrm{b}_{1} \mathrm{x}+\mathrm{c}_{1}$. The second order polynomial is found to be $y=0.21 x^{2}-15.99 x+$ 309.45 with error in the coefficient $0.02,1.12,9.25$ respectively. The standard deviation $(\sigma)$ for the second order polynomial model is 17.75 .

Variation of relative flux density along line 12:

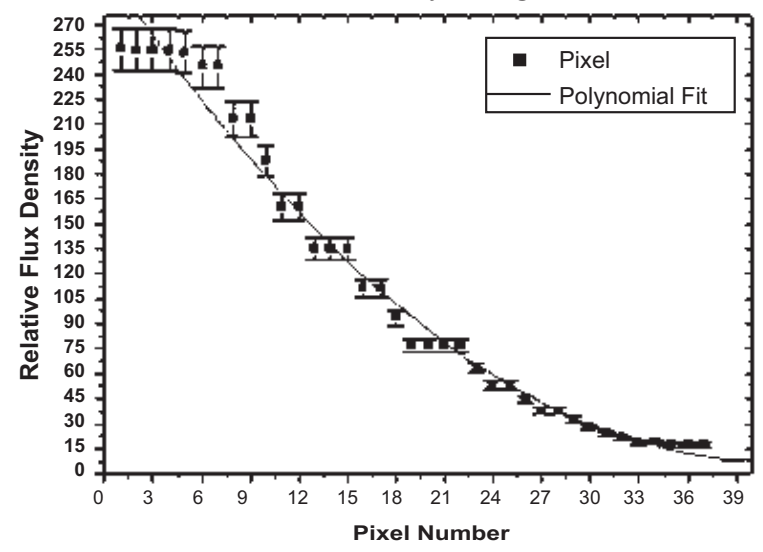

Fig 14: variation of relative flux density along line twelve

The variation of relative flux density along the line twelve are shown in figure 14. The graph are plotted number or pixel versus relative flux density .The graph show that relative flux density continuously decreases from inner to outer regions. The nature of relationship between relative flux density and pixel number is determined by using parabolic fit model $y=a_{1} x^{2}+b_{1} x$ $+c_{1}$. The curve fitting of relative flux density against 
pixel number for line twelve is shown in Fig. 14 where a solid curve represents second order polynomial fit. For the line twelve, the second order polynomial is found to be $y=0.17 x^{2}-14.41 x+304.73$ with error in the coefficient $0.02,0.78,6.56$ respectively. The standard deviation $(\sigma)$ for the second order polynomial model is 12.74. While comparing standard error with standard deviation obtained from second order polynomial fit, standard error is less than standard deviation which shows that the relative flux density follows the second order polynomial fit.

\section{Variation of Flux Density in the Outer Regions}

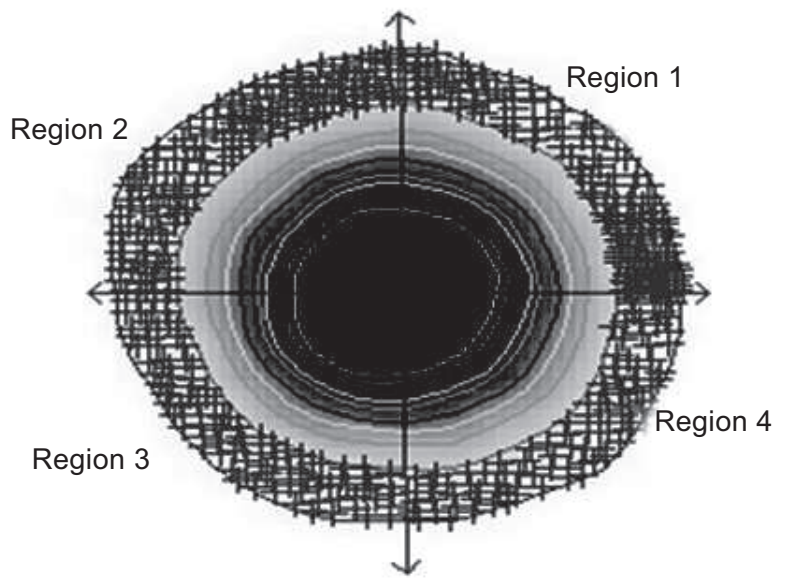

Fig 15: variation of flux density in the outer region of Sirius

We have studied the relative flux density in outer regions, for this we have divided the image into four quadrants. First column of Table 3, shows quadrant i.e., region second and third column represents number of pixel and total flux density and last column represents flux density per pixel.

\begin{tabular}{llll}
\hline Quadrant & $\mathrm{N}$ & $\sum \mathrm{F}_{\mathrm{D}}$ & $\mathrm{F}_{\mathrm{DP}}$ \\
\hline First & 256 & 5934.00 & 23.17 \\
Second & 461 & 10862.65 & 23.56 \\
Third & 338 & 7829.12 & 23.16 \\
Fourth & 200 & 4621.12 & 23.10 \\
\hline
\end{tabular}

The variation of flux density per pixel is found almost constant for each quadrant however, flux density per pixel is found slightly more in second quadrant with compare to other quadrants.

The nature of image of Sirius is found to be symmetric in outer regions, so no flatting disk is observed in our image analysis.

\section{CONCLUSION}

The main sequence star Sirius was observed from National Observatory Nagarkot, Nepal during the period of December 2011 to November 2012, through 16 inch LX200GPS Schmidt- Cassegrain Telescope using AUTOSTAR SUIT 3.08 software and image was processed in software ALADIN 2.5. Our conclusion of dissertation work are as follows:

- The inner surface of star shows homogeneous relative flux density. The values of relative flux density in the region of interest ranges from 15.75 to 255.05 and relative flux density per pixel ranges from 84.82 to 116.73. There was consistent decrease in relative flux density from inner to outer regions of interest of surface of the star Sirius. The variation of relative flux density from inner to outer region may be ongoing process of hydrogen burning in the core.

- There were no observed asymmetry in the star in our analysis of image.

- No significant flatting was observed from our analysis which matched with works of Royer, F., et al. And Kervella, P., et al. ${ }^{[4],[5]}$

- The standard error in Relative Flux Density $\mathrm{F}_{\mathrm{D}(\mathrm{Er} \pm)}$ was less than standard deviation $(\sigma)$ obtained from second order polynomial fit. The variation of relative flux density from inner to outer region follows the second order polynomial, implying that it was the best method to leak the radiation produced in the core of the star as far as possible.

\section{ACKNOWLEDGEMENT}

We acknowledge B.P. Koirala Memorial Planetarium, Observatory and Science Museum Development Board, Ministry of Science, Technology \& Environment. Government of Nepal for allotting several nights for the observation. One of the authors (Birendra Prasad Pant) acknowledges the board for financial support to carry out the dissertation work.

\section{REFRENCES}

[1] van Leeuwen, F. November. 2007. "Validation of the new Hipparcos reduction”. A\&A. 474 (2): 653-664

[2] Sky and Telescope. April. 1998. Based on computations from Hipparcos data. p:60

[3] Schaaf, Fred. 2008. The Brightest Stars. Hoboken, New Jersey: John Wiley \& Sons. p. 94

[4] Royer, F., et al. 2002. "Rotational velocities of A-type stars. I. Measurement of v sin $\mathrm{i}$ in the southern hemisphere". A \& A. 381 (1): 105-121.

[5] Kervella, P., et al. 2003. "The interferometric diameter and internal structure of Sirius A". A\&A. 407 (2): 681-688 